\title{
The influence of limited visual sensing on the Reynolds flocking algorithm
}

\author{
Enrica Soria, Student Member, IEEE, Fabrizio Schiano, Member, IEEE, \\ and Dario Floreano, Senior Member, IEEE
}

\begin{abstract}
The interest in multi-drone systems flourished in the last decade and their application is promising in many fields. We believe that in order to make drone swarms flying smoothly and reliably in real-world scenarios we need a first intermediate step which consists in the analysis of the effects of limited sensing on the behavior of the swarm. In nature, the central sensor modality often used for achieving flocking is vision. In this work, we study how the reduction in the field of view and the orientation of the visual sensors affect the performance of the Reynolds flocking algorithm used to control the swarm. To quantify the impact of limited visual sensing, we introduce different metrics such as (i) order, (ii) safety, (iii) union and (iv) connectivity. As Nature suggests, our results confirm that lateral vision is essential for coordinating the movements of the individuals. Moreover, the analysis we provide will simplify the tuning of the Reynolds flocking algorithm which is crucial for real-world deployment and, especially for aerial swarms, it depends on the envisioned application. We achieve the results presented in this paper through extensive Monte-Carlo simulations and integrate them with the use of genetic algorithm optimization.
\end{abstract}

\section{INTRODUCTION}

Recent advances in the field of aerial robotics and sensor technologies have greatly enhanced the capabilities of unmanned aerial vehicles (UAVs). One consequence of this outcome has been the growing interest in multi-aerial vehicle applications [1], [2], [3]. Clear benefits of multi-drone systems are envisioned for a wide range of missions including search and rescue [4], long-term monitoring [5], sensor data collection [6], indoor navigation [7], environment exploration [8] and cooperative grasping and transportation [9]. There are numerous advantages in using multiple drones instead of a single one, such as the coverage of larger areas, the possibility of reducing the total time of the mission and the ability to perform tasks which would be unfeasible for a single drone. These are merely a few of the key positive aspects associated with the use of multi-drone systems. However, an increase in the size of the system is also accompanied by an increase in its complexity. Today, the high complexity of multi-drone systems is commonly addressed by relying on a centralized approach [9], [10], [11]. These robotic systems mostly use an external computing unit that depends on centralized systems (e.g., motion capture system or GNSS) and other sensory information gathered by the agents of the swarm. Based on this information, the central unit generates and communicates the control commands to the whole group. Several studies [12], [13], [14], [15], [16], [17], [18] highlighted that often biological collectives do not work in this manner. Indeed, the approach used by animals such as ants, bees, and birds is generally decentralized and distributed among the individuals of the group. In particular, one behavior exhibited by animals that drives significant research efforts these days is flocking [19], [20], the coordinated collective motion that several flying species manifest. Flocking is considered as an emergent phenomenon since it arises from the ensemble of each individual's decisions and is based only on local information. Swarm robotics can significantly benefit from taking inspiration from the behavior of these biological systems. For example, a decentralized and distributed approach can make the system easily scalable and robust to failures. For this reason, the implementation of swarms based on these principles has received a lot of attention in recent years [21], [22].

The well-known Reynolds algorithm [19] offers a way of describing the global motion of a flock through three rules acting on each agent simultaneously. In this work, we will refer to them as (a) alignment, the tendency to steer the agents towards the average heading of their neighbors, (b) cohesion, the tendency to steer the agents towards the swarm center, and (c) separation, the tendency to drive away the agents from their neighbors to avoid collisions.

However, when implementing flocking algorithms it becomes clear that their tuning is highly dependent on the application. Indeed, in [23] the authors use evolutionary algorithms to find the optimal configuration of parameters for the navigation task of their flock. In some applications, the central interest can be to remain connected [24], [25], while in others, it may be more crucial to control the number of subgroups that originate during the operation. An example of the second case is patrolling [26], where the flock is allowed to split, but a minimum number of agents per cluster is needed to enable stereo vision.

Another evidence from nature is that several animals rely on visual information to achieve navigation, collision avoidance, and remain connected with the other individuals of the group [27], [28], [29], [30]. Using vision as the main sensor modality for the coordination of robotic swarms presents several advantages. First of all, a visual sensor combined with an Inertial Measurement Unit (IMU) represent the minimum sensor configuration for achieving autonomous navigation in complex environments. Moreover, the camera is a passive sensor and therefore requires less energy than active sensors (e.g. lidar and sonar). A drawback of relying on vision is that the detection of far objects can be very challenging. However, in dense swarms, we can assume that the agents are sufficiently 
close to be perceived. Although the visual organs of birds are limited in range and angular span, these animals can fly in perfect choreographed synchronization. Sensor limitations are seldom taken into account in robotics since they can be hard to model [31]. However, understanding their effects can be fundamental to bring aerial swarms from lab-conditions to real-world scenarios.

The visual processes of animals include several aspects. In particular, a distinction can be made between physical and perceptual properties. Examples of the former include the geometric field that the eyes can perceive, their rapidity to adapt to light and environmental changes and the range of the detected light wavelengths. The visual perception, instead, is defined as the ability of animals to assimilate information from the surroundings and it is an open field of research for psychologists, neuroscientists, and molecular biologists.

In this paper, we focus on analyzing the impact of the physical geometric properties of visual sensing, specifically the width angle of the field of view (FOV) and its orientation, on the performance of a Reynolds flock. Because the aimed behavior of a swarm is often application-dependent, we quantify the effects of the two aforementioned visual properties with different metrics that can be relevant to different scenarios. Since the same limitations exist for the cameras that are equipped on the majority of off-the-shelf commercial drones, we believe that this work can represent an essential step for future robotics developments.

The rest of the paper is organized as follows. Sect. II reviews the notation, the concepts of limited sensing and the Reynolds flocking model. Sect. III defines the metrics which will be applied to evaluate the effects of limited visual sensing. Finally, Sect. IV presents the results of our work, followed by Sect. V that concludes the paper and outlines possible future directions.

\section{PReliminaries}

In this work we consider a set of $N$ point-mass agents labeled by $i \in\{1,2,3, \ldots, N\}$. The position, velocity and acceleration of the agent $i$ are denoted by $\boldsymbol{x}_{i}, \dot{\boldsymbol{x}}_{i}, \ddot{\boldsymbol{x}}_{i} \in \mathbb{R}^{2}$, respectively. In order to keep our notation concise we let $d_{i j}=\left\|\boldsymbol{x}_{j}-\boldsymbol{x}_{i}\right\|$ where $\|\cdot\|$ denotes the Euclidean norm, and $\overline{\boldsymbol{x}}_{i j}=\boldsymbol{x}_{i j} / d_{i j}^{2}$. We then let $\boldsymbol{I}_{N} \in \mathbb{R}^{N \times N}$ represent the identity matrix of dimension $N, \mathbf{0}_{N} \in \mathbb{R}^{N}$ a vector of all zeros and $\mathbf{0}_{N \times N} \in \mathbb{R}^{N \times N}$ a matrix of all zeros. The operator $\operatorname{diag}(\cdot)$ returns a square diagonal matrix with the elements of the input vector on the main diagonal and the operator $\operatorname{stack}(\cdot)$ returns a matrix containing a vertical stacking of the arguments.

We model the swarm with a directed sensing graph $\mathcal{G}=$ $(\mathcal{V}, \mathcal{E})$, where the vertex set $\mathcal{V}=\{1 \ldots N\}$ represents the agents, and the edge set $\mathcal{E} \subseteq \mathcal{V} \times \mathcal{V}$ contains the pairs of agents $(i, j) \in \mathcal{E}$ for which agent $i$ can sense agent $j$. We denote as $\mathcal{N}_{i}=\{j \in \mathcal{V} \mid(i, j) \in \mathcal{E}\} \subset \mathcal{V}$ the set of neighbors of an agent $i$ in $\mathcal{G}$ and as $\left|\mathcal{N}_{i}\right|$ its cardinality ${ }^{1}$. We also define

\footnotetext{
${ }^{1}$ Note that both the set of edges $\mathcal{E}$ and the one of neighbors $\mathcal{N}_{i}$ of a specific agent $i$ are time-varying. However, we will omit their time dependency throughout the paper for brevity.
}

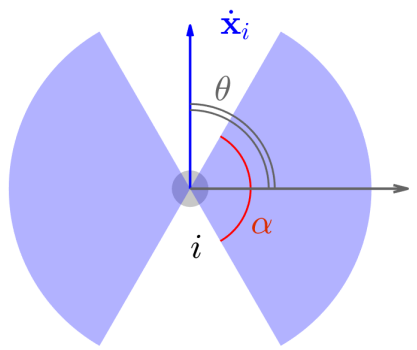

Fig. 1: Illustration of the width and azimuth angles, $\alpha$ and $\theta$. In particular, this configuration is associated with values $\alpha=120^{\circ}$ and $\theta=90^{\circ}$.

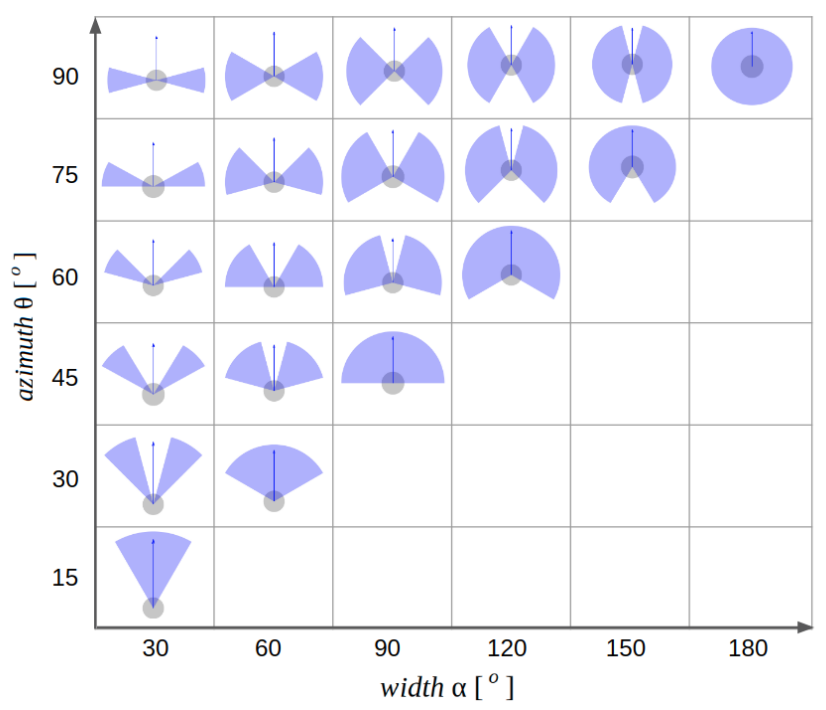

Fig. 2: Graphical representation of the sensing configurations of an agent for different values of width $\alpha$ and azimuth $\theta$. Note that the positive values on the $\theta$-axis refer to the 'right eye', while respective values for the 'left eye' have a negative sign for the hypothesis of symmetry that we assume. When $\theta=\alpha / 2$ the two sensed regions overlap on one edge and merge in a unique circular sector. For every other azimuth value, the agent perceives two non-intersecting sectors and for $\theta=90^{\circ}$ (first row) the eyes face opposite directions.

$\delta_{i j}$, a function of two agents $i$ and $j$, which takes value 0 if $j \notin \mathcal{N}_{i}$ and 1 if $j \in \mathcal{N}_{i}$. Another concept borrowed from algebraic graph theory is algebraic connectivity [32], also known as connectivity eigenvalue. This is the second smallest eigenvalue of the Laplacian matrix [32] associated with the undirected graph $\mathcal{G}^{\prime}$ obtained from $\mathcal{G}$ and it is usually denoted by $\lambda_{2}$. The algebraic connectivity has been extensively used in swarm robotics [24], [25] because the magnitude of this value reflects crucial qualities of the graph. However, its mathematical details are beyond the scope of this paper.

\section{A. Limited field of view}

In this paper we study the influence of the geometric visual constraints on the ability of flocking. For the sake of an easier 


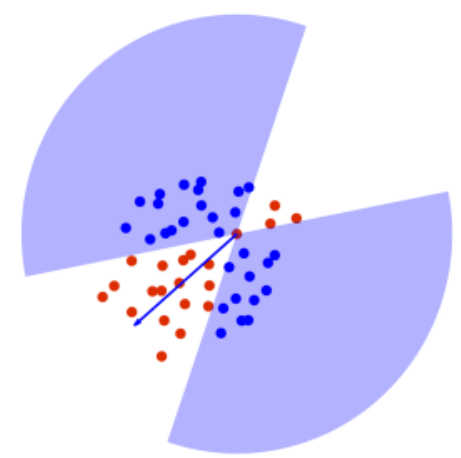

Fig. 3: Schema of a Reynolds flock where the FOV of one agent $i$ is highlighted. The agent has a visual sector of width $\alpha=120^{\circ}$ and azimuth $\theta=90^{\circ}$. The flock members in blue are the ones perceived by $i$ (the neighbors $\mathcal{N}_{i}$ ), while the unperceived members are in red.

visualization and interpretability of the results, we choose to implement our model in two dimensions. We endow every agent with two eyes, each having sight over a portion of the surroundings, i.e. a circular sector centered on the agent's position. The eyes are symmetrically placed about the direction of the agent's velocity. Since we are interested in dense swarms, we assume that the radius of perception is bigger than the size of the flock, therefore we do not add a constraint on the visual range. To model the described configuration, we define two distinct parameters:

- the FOV width $(\alpha)$ is the angular span that an eye can detect and it ranges from $0^{\circ}$ to $180^{\circ}$. On a UAV this would correspond to the FOV of an on-board camera;

- the FOV azimuth $(\theta)$ describes the visual direction of the eye and it varies from $\alpha / 2$ to $90^{\circ}$. The choice to limit $\theta$ to $90^{\circ}$ is justified by the analogy with the orientation of the birds eyes [33]. On a UAV this would correspond to the angle at which the camera centre is placed w.r.t. the $x$-axis of the body-frame of the robot.

Fig. 1 provides an illustration for the two parameters, while Fig. 2 shows different configurations of the FOV for varying values of width $\alpha$ and azimuth $\theta$.

Because of its limited FOV, an agent $i$ can only sense a portion of the surrounding space. This defines the set of flock members that $i$ can perceive at time $t$, namely its neighborhood $\mathcal{N}_{i}$. Fig. 3 illustrates an example of the neighborhood of $i$, for a sensor configuration of $\alpha=120^{\circ}$ and $\theta=90^{\circ}$.

\section{B. Reynolds flocking model}

According to the Reynolds model [19], the motion of every agent in the flock is defined by the three rules of alignment, cohesion and separation, weighted by the constant coefficients $c_{a}, c_{c}$, and $c_{s}$, respectively. In the following, we will also refer to them as Reynolds gains.

The acceleration of agent $i$ is determined by its neighbors $\mathcal{N}_{i}$ as follows:

$$
\begin{aligned}
\ddot{\boldsymbol{x}}_{i}=\underbrace{\frac{c_{a}}{\left|\mathcal{N}_{i}\right|} \sum_{j \neq i} \dot{\boldsymbol{x}}_{j}-\dot{\boldsymbol{x}}_{i}}_{\text {alignment }}+\underbrace{\frac{c_{c}}{\left|\mathcal{N}_{i}\right|} \sum_{j \neq i} \boldsymbol{x}_{j}-\boldsymbol{x}_{i}+}_{\text {cohesion }} & \\
& -\underbrace{\frac{c_{s}}{\left|\mathcal{N}_{i}\right|} \sum_{j \neq i} \frac{\boldsymbol{x}_{j}-\boldsymbol{x}_{i}}{\| \boldsymbol{x}_{j}-\left.\boldsymbol{x}_{i}\right|^{2}}}_{\text {separation }}
\end{aligned}
$$

The choice of the gain values in the Reynolds flocking is not unique and in many situations it is application-dependent. For instance, in operations of maximal area coverage, increasing the separation gain could help to amplify the spreading of the drones. Instead, in operations that require the flock to squeeze through narrow canyons, the cohesion could be increased to make the group fit into a reduced space.

We restrict the gain values to the range $[1,10]$. Notice that a separation gain equal to $1, c_{s}=1$, means that whenever two agent are situated at $1 \mathrm{~m}$ of distance from each other, they are repulsed from each other with an acceleration of magnitude $1 \mathrm{~m} / \mathrm{s}^{2}$. Similar considerations can be done for the other gains.

Let us denote $\boldsymbol{x}=\operatorname{stack}\left(\boldsymbol{x}_{1}, \boldsymbol{x}_{2}, \cdots \boldsymbol{x}_{N}\right), \quad \dot{\boldsymbol{x}}=$ $\operatorname{stack}\left(\dot{\boldsymbol{x}}_{1}, \dot{\boldsymbol{x}}_{2}, \cdots \dot{\boldsymbol{x}}_{N}\right), \ddot{\boldsymbol{x}}=\operatorname{stack}\left(\ddot{\boldsymbol{x}}_{1}, \ddot{\boldsymbol{x}}_{2}, \cdots \ddot{\boldsymbol{x}}_{N}\right) \in \mathbb{R}^{2 N}$ respectively containing the positions, velocities, and accelerations of the agents of the swarm. The second-order system defining the motion of the flock can be written as:

$$
\ddot{\boldsymbol{x}}=c_{a} \boldsymbol{A} \dot{\boldsymbol{x}}+c_{c} \boldsymbol{A} \boldsymbol{x}-c_{s} \boldsymbol{H}
$$

where $\boldsymbol{A} \in \mathbb{R}^{2 N \times 2 N}$ is a matrix composed by blocks $\boldsymbol{A}_{i j}=$ $\left(\delta_{i j} /\left|\mathcal{N}_{i}\right|\right) \boldsymbol{I}_{2} \in \mathbb{R}^{2 \times 2}$ if $i \neq j$ and $\boldsymbol{A}_{i j}=-\boldsymbol{I}_{2} \in \mathbb{R}^{2 \times 2}$ if $i=j$, and $\boldsymbol{H} \in \mathbb{R}^{2 N \times 2 N}$ is composed by blocks $\boldsymbol{H}_{i j}=$ $\left(1 /\left|\mathcal{N}_{i}\right|\right) \operatorname{diag}\left(\overline{\boldsymbol{x}}_{i j}\right) \in \mathbb{R}^{2 \times 2}$ if $i \neq j$ and $\boldsymbol{H}_{i j}=\mathbf{0}_{2 \times 2}$ if $i=j$.

\section{Agents dynamics}

In our simulations, the dynamics of the agents is reproduced in discrete time according to a double integrator model [34]. For every time step $t_{k}=k \cdot d t, k \in\{1,2,3 \ldots, K\}$ and every agent $i$, it holds

$$
\begin{aligned}
\ddot{\boldsymbol{x}}_{i}^{k} & =\boldsymbol{u}_{i}^{k} \\
\dot{\boldsymbol{x}}_{i}^{k} & =\dot{\boldsymbol{x}}_{i}^{k-1}+\ddot{\boldsymbol{x}}_{i}^{k} d t \\
\boldsymbol{x}_{i}^{k} & =\boldsymbol{x}_{i}^{k-1}+\dot{\boldsymbol{x}}_{i}^{k} d t
\end{aligned}
$$

where $d t$ represents the step used for the temporal discretization of the system, and $\boldsymbol{x}_{i}^{k}, \dot{\boldsymbol{x}}_{i}^{k} \ddot{\boldsymbol{x}}_{i}^{k} \in \mathbb{R}^{2}$ are the position, velocity and acceleration of the $i$-th agent at time $t_{k}$, respectively. The agents update their motion following the Reynolds rules described in Sect. II-B. In this respect, the Reynolds accelerations are the control inputs $\boldsymbol{u}_{i}^{k} \in \mathbb{R}^{2}$.

To narrow the gap between simulation and reality we consider physical constraints on the magnitudes of velocities and accelerations, expressed by $\left\|\dot{\boldsymbol{x}}_{i}^{k}\right\| \leq \bar{v}$ and $\left\|\ddot{\boldsymbol{x}}_{i}^{k}\right\| \leq \bar{a}$. 

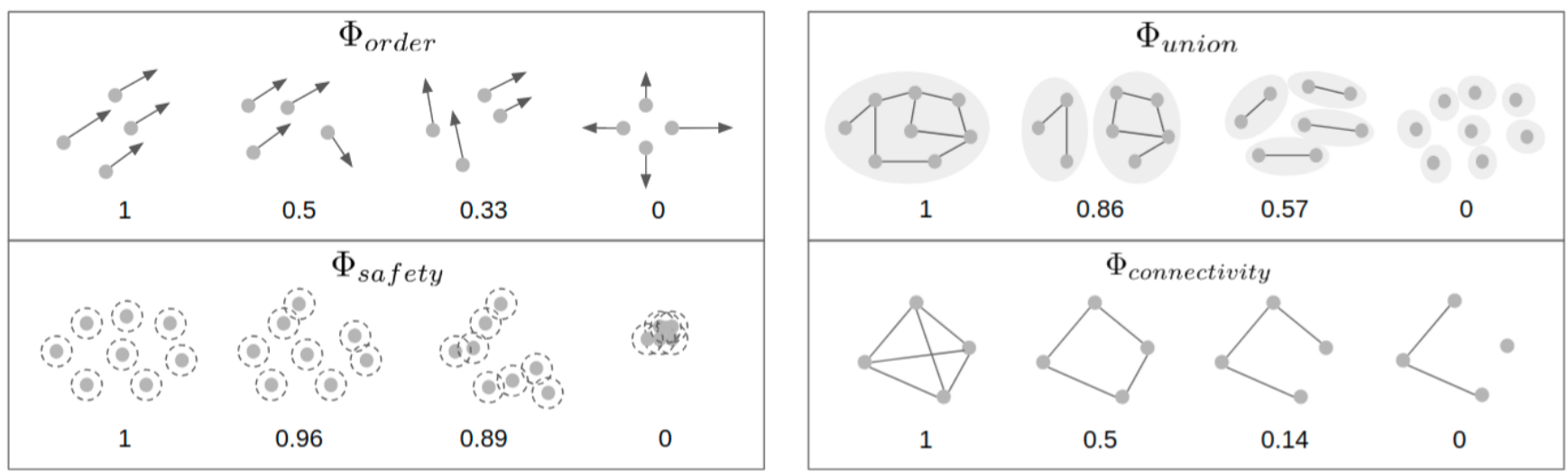

Fig. 4: This figure illustrates different flock configurations for varying values of the order $\left(\Phi_{\text {order }}\right)$, safety $\left(\Phi_{\text {safety }}\right)$, union $\left(\Phi_{\text {union }}\right)$ and connectivity $\left(\Phi_{\text {connectivity }}\right)$ metrics. In general, 1 corresponds to a high score, while 0 refers to a poor score. Significant levels are chosen for every metric. In particular, for the safety metric, important changes in the flock configuration already happen in the highest half-range.

\section{FLOCKING PERFORMANCE METRICS}

When limiting the FOV, the guarantee that the agents form a single group where all components move at the same speed in the same direction does not hold anymore. Indeed, two agents that do not perceive each other act independently. Under this condition, the flock can split in multiple subgroups that do not affect each others movements, and collisions can occur. To evaluate the collective performance, four relevant metrics are introduced. Several metrics for describing the correlation of the agents' movements and the collision risk have been adopted in the literature for describing both robotic systems and animal groups [23], [35], [15]. Furthermore, in swarm robotics, the algebraic connectivity has proven to be useful in many applications, especially the ones where a flow of information has to be ensured among all the robots of the swarm (e.g., exploration and target tracking). By taking into account a metric directly related to this quantity we aim to make our analysis relevant also for those users who are interested in maintaining the connectivity of the graph while flocking with limited sensors. In this work all metrics are scaled to be equal to 1 in the best-case scenario. Nominally, they are:

- the order metric, $\Phi_{\text {order }}^{k}$ : it captures the correlation of the agents' movements and gives an indication about how ordered the flock is. At every temporal instant $t_{k}$, it is expressed by

$$
\Phi_{\text {order }}^{k}=\frac{1}{N(N-1)} \sum_{i, j \neq i} \frac{\dot{\boldsymbol{x}}_{i}^{k} \cdot \dot{\boldsymbol{x}}_{j}^{k}}{\left\|\dot{\boldsymbol{x}}_{i}^{k}\right\|\left\|\dot{\boldsymbol{x}}_{i}^{k}\right\|} .
$$

- The safety metric, $\Phi_{\text {safety }}^{k}$ : it measures the risk of collisions among the members of the flock at a given temporal instant. We define $r_{s}$ as the radius of a virtual area that surrounds the agent where the presence of other agents should be avoided for safety reasons. For real UAVs, it corresponds to the dimension of the robot, plus an arbitrary margin which is higher in more conservative approaches. The number of violations is $n_{s}=$ $\mid\left\{(i, j)\right.$ s.t. $\left.j \neq i \wedge d_{i j}<r_{s}\right\} \mid$. Therefore, at time $t_{k}$ the metric can be expressed as

$$
\Phi_{\text {safety }}^{k}=1-\frac{n_{s}}{N(N-1)} .
$$

- The union metric, $\Phi_{\text {union }}^{k}$ : it reflects how scattered the group members are and it counts the number of independent subgroups that originates along the simulation. We define $n_{c}$ as the number of connected components of the undirected graph that corresponds to the flock topology, then at time $t_{k}$ it holds

$$
\Phi_{\text {union }}^{k}=1-\frac{n_{c}-1}{N-1} .
$$

- The connectivity metric, $\Phi_{\text {connectivity }}^{k}$ it is defined from the algebraic connectivity of the graph corresponding to the flock configuration. We define the value of the metric at time $t_{k}$ as

$$
\Phi_{\text {connectivity }}^{k}=\frac{\lambda_{2}}{N}
$$

where $\lambda_{2}$ is defined in Sect. II. Notice that $\Phi_{\text {connectivity }}^{k} \neq 0$ only when $\Phi_{\text {union }}^{k}=1$. In this sense, the connectivity metric is complementary to the union metric.

To evaluate the global performance of the flock during the time $T$ of a simulation, the metrics that have been defined above for a generic time instant $t_{k}$ are averaged over the time window. Then, the order metric of the flock referred to a simulation of length $T$ is

$$
\Phi_{\text {order }}=\frac{\sum_{k=1}^{K} \Phi_{\text {order }}^{k}}{K}
$$

and the same holds for the other metrics. 


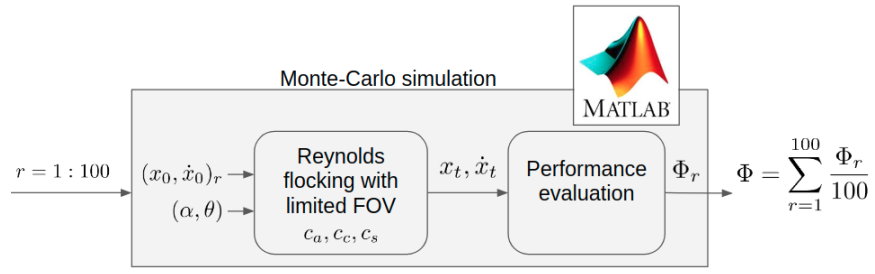

Fig. 5: Schema of the simulation workflow used for testing a given sensor configuration $(\alpha, \theta)$ in Reynolds flocking with gains $c_{a}, c_{c}$ and $c_{s}$. The outputs of the workflow are the performance metrics $\Phi_{\text {order }}, \Phi_{\text {safety }}, \Phi_{\text {union }}$ and $\Phi_{\text {connectivity }}$, averaged over the $r=100$ repetitions of the Monte-Carlo method.

\section{Simulation Results}

In this section, we present the results about the effects of limited visual sensing (modeled using the two parameters of width and azimuth introduced in Sect. II-A) on the flocking performance metrics described in Sect. III. An extensive analysis of their influence is carried out by running the Reynolds algorithm at different sensor configurations.

Besides depending on the visual configuration, the performance results also vary w.r.t. the Reynolds gains $c_{a}, c_{c}$ and $c_{s}$. As we mentioned in Sect. II-A, the choice of the right combination of those constants can be application-dependent and, therefore, non-unique. Consequently, we study how the performance metrics vary according to this choice.

In the first place, we select fixed triplets for the Reynolds gains and we analyze the metrics when the alignment, the cohesion or the separation effect are privileged, once at a time. An additional case is studied for equal values of the gains.

Another approach can be preferred when we are aware of the metric w.r.t. our flock should be optimized. Therefore, in a subsequent study we analyze the results for Reynolds gains that are optimized for each of the metrics considered, one at a time. Genetic algorithms are applied to find the optimal flocking parameters. In the following, we present the simulation setup we used.

All simulations have been run in MATLAB R2017b. The work-flow we used is summarized in Fig. 5. Specifically, we consider a swarm of $N=50$ agents. The width angle $\alpha$ is varied with constant steps of $10^{\circ}$ in the range $\left[10^{\circ}, 180^{\circ}\right]$, while $\theta$ is increased with steps of $5^{\circ}$ in the range $\left[\alpha / 2,90^{\circ}\right]$. For each sensor configuration $(\alpha, \theta)$ we apply a Monte-Carlo method that repeatedly selects random samples of the initial conditions for the flock, i.e. initial positions and velocities of the agents $\left(\boldsymbol{x}_{0}, \dot{\boldsymbol{x}}_{0}\right)$. A batch of 100 simulations is run, and the final score is the average over the batch.

At $t_{0}=0 s$ the agents are initialized with random positions and velocities. The former follow a uniform distribution over a cube of $10 \mathrm{~m}$ edge, while the latter obey a multi-normal law with mean $\mathbf{0}_{2 N} \mathrm{~m} / \mathrm{s}$ and covariance $3 \boldsymbol{I}_{2 N} \mathrm{~m}^{2} / \mathrm{s}^{2}$. The discretization time step used in simulation is $\delta t=0.05 s$ and the total time is $T=50 \mathrm{~s}$.

\section{A. Fixed Reynolds coefficients}

In this section we evaluate the flocking properties when the Reynolds gains are fixed to triplets that privilege one effect over the others: alignment, cohesion or separation. Finally, a triplet with equal gains is evaluated. The values applied in our simulations as summarized in table I.

TABLE I: List of the different Reynolds gain triplets used in simulation.

\begin{tabular}{ccc}
\hline$c_{a}$ & $c_{c}$ & $c_{s}$ \\
\hline 10 & 1 & 1 \\
1 & 10 & 1 \\
1 & 1 & 10 \\
1 & 1 & 1 \\
\hline
\end{tabular}

As a general consideration, we may reasonably expect that a decline in the performance of the flock is proportional to a diminution of the width angle $\alpha$. Indeed, a decrease in the visual angle of the agents determines a reduction in the information that they can capture from the surroundings. This tendency is generally confirmed by the union and the connectivity metrics for all the choices of gains, $(I-I V, C)$ and $(I-I V, D)$ in table III. Instead, this is not the case for the order and the safety metrics, $(I-I V, A)$ and $(I-I V, B)$, for which the trends shown are more complex.

If we observe the order metric $\Phi_{\text {order }},(I-I V, A)$, it is immediately noticeable that the configurations on the halfdiagonals (from top left to the centre) perform better than the average (the yellow color in the figures corresponds to high scores), and this fact is independent of the choice of gains. Fig. 6 presents a graphical excerpt of the sensor configurations corresponding to these diagonal regions, from which we can infer the visual portions that they have in common: two thin lateral sectors. High values in order metric are also present in the upper regions of the figures, where the azimuth angle is $\theta=90^{\circ}$. Again, this holds for every choice of the flocking gains. These upper regions correspond to visual configurations with diametrically opposed sensors, having sight on the lateral sides. These results corroborate the hypothesis that lateral vision is important in biology, especially for those species exhibiting collective motion. Indeed, the majority of birds in nature presents a narrow binocular sector that varies on average between $20^{\circ}$ and $30^{\circ}$ and a wide FOV that covers well the two lateral regions [33].

The results about the safety metric $\Phi_{\text {safety }}$ are highly dependent on the Reynolds gains. In fact, in $(I-I V, B)$ we can observe very different patterns. Moreover, it can be noticed that this metric takes values in a very limited range. This is partly due to the normalization factor, that counts the number of all possible pairs of agents. Therefore, for a large swarm the addition of one collision would correspond to a very limited decrease in the safety value. As intuition suggests, our results confirm that the safety metric deteriorates when the cohesion gain increases, see $(I I, B)$. However, unexpectedly, the lowest safety values correspond to the biggest FOV widths. This can be explained by referring to the union metric $\Phi_{\text {union }}$ in $(I I, C)$. 


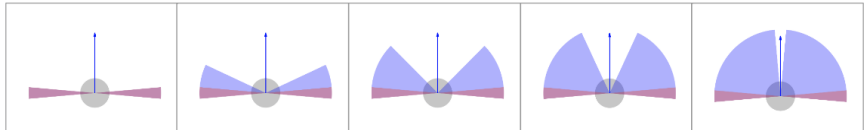

Fig. 6: Configurations of the FOV corresponding to the left half-diagonal of the $(\alpha, \theta)$-space (from top left to the centre). This configurations are associated with high scores in the order metric. In particular, from left to right, they are: $(\alpha=10, \theta=90),(30,80),(50,70),(70,60)$ and $(90,50)$, where the width and azimuth angles are expressed in degrees $\left.{ }^{\circ}\right]$. The intersection between all of them is highlighted in red.

High values of $\Phi_{\text {union }}$ correspond to the tendency of the agents to create a unique group, thereby increasing the chance of collision.

One non-trivial remark about the union metric in $(I I I, C)$, is that for very small values of width $\alpha$ the values of the azimuth $\theta$ performing the best are the extreme ones, either close to $0^{\circ}$ or to $90^{\circ}$. The explanation of this phenomenon involves more analysis on the system and it will be part of our future work.

As anticipated, a non-zero value of the connectivity metric $\Phi_{\text {connectivity }}$ is only possible when $\Phi_{\text {union }}^{k}$ takes the maximum value, 1 , at least at some time instants. During a simulation, the flock may split and rejoin. In such instances, the instantaneous connectivity metric $\Phi_{\text {connectivity }}^{k}$ passes from positive to null values and vice versa, and the global value of $\Phi_{\text {connectivity }}$ is the average over time. Globally, we notice that the larger the width angle, the higher the score.

\section{B. Optimized Reynolds coefficients}

The approach of finding optimal flocking parameters through genetic algorithms has been applied in previous work [23] and here it is justified by the non-convexity of the relationship between the parameters and the performance. A grid search method could also be applied although testing all the gain triplets would require a longer simulation time.

In table II we resume the parameters used in the genetic algorithm.

TABLE II: Parameters of the genetic algorithm used to determine the optimal values of the Reynolds gains. The algorithm is part of the built-in functions in MATLAB. The optimization is performed w.r.t. each of the metric functions and for every sensor configuration $(\alpha, \theta)$.

\begin{tabular}{cc}
\hline Parameter & Value \\
\hline Variables & $c_{a}, c_{c}, c_{s}$ \\
Range for the variables & {$[1,10] \times[1,10] \times[1,10] \in \mathbb{R}^{3}$} \\
Fitness function & $\Phi_{\text {order }}, \Phi_{\text {safety }}, \Phi_{\text {union }}, \Phi_{\text {connectivity }}$ \\
Population size & 10 \\
Number of generations & 10 \\
Scaling operator & 'proportional' \\
Selection operator & 'tournament' \\
Crossover operator & 'scattered' \\
Mutation operator & 'gaussian' \\
\hline
\end{tabular}

Row $(V)$ in table III shows the results of the four met- rics associated with the flocking simulations with optimized parameters. One consideration is that the plots display more discontinuities over the configuration-space $(\alpha, \theta)$ compared to the previous results. A motivation for this is that genetic algorithms rely on a stochastic approach and they do not guarantee the convergence to the global optima. In addition, to keep the total simulation time affordable, our optimization algorithm involved a reduced number of individuals and generations.

In general, the trends highlighted in Sect. IV-A are confirmed in this section. Indeed, similarly to the previous cases, the order metric $\Phi_{\text {order }}$ shows the best performing values on the diagonal and in the upper area. To a more accurate analysis, it seems that in the diagonal region the genetic algorithm led to some improvements, i.e. the yellow portion in $(V, A)$ is wider. The safety metric $\Phi_{\text {safety }}$ globally presents high values, with some outliers in the upper region, where poorer results were already present for fixed Reynolds gains. The union metric $\Phi_{\text {union }}$ varies in a smaller range, indicating a global enhancement. Finally, the connectivity metric $\Phi_{\text {connectivity }}$ does not display substantial modifications and presents positive scores only on the right corner where the width angle is wide.

\section{CONCLUSIONS AND FUTURE WORK}

In this work, we presented a numerical analysis about the impact of limitations in visual sensing on flocking systems, from the perspective of different and complementary performance metrics.

We believe that the results presented in this paper can be a starting point for filling the gap between simulations and reality when implementing flocking algorithms. Moreover, this analysis could be used to solve the problem of optimal sensor placement, when the application of the flocking system is known a priori and a choice of the sensors and their orientation has to be made.

The results presented are promising and create new avenues of research that are worth investigating. More complex models should be taken into account to observe the effects of different drone dynamics on the Reynolds flocking, e.g. fixed-wing or quadrotor drones and how the sensory limitations affect their flight in swarms. Moreover, noise modeling and delays should be taken into account to narrow the gap between simulation and reality.

In addition to the limited FOV, we would like to consider other limitations of the visual sensors such as limited range and possible occlusions generated by other agents [31]. Indeed, in the real-world, visual perception is influenced by the distance. Higher definition measurements are obtained for nearby neighbours and worse for far-away ones. Moreover, every object detected by a camera generates a blind cone behind it, occluding the view over a part of the surrounding space. Finally, in order to make our approach more realistic we think that is important to add obstacles along the navigation path and analyze how the behavior of the flock adapts.

\section{ACKNOWLEDGMENT}

The authors would like to thank Fabian Schilling, Julien Lecoeur, Vivek Ramachandran and Anthony De Bortoli for 
(A) $\Phi_{\text {order }}$
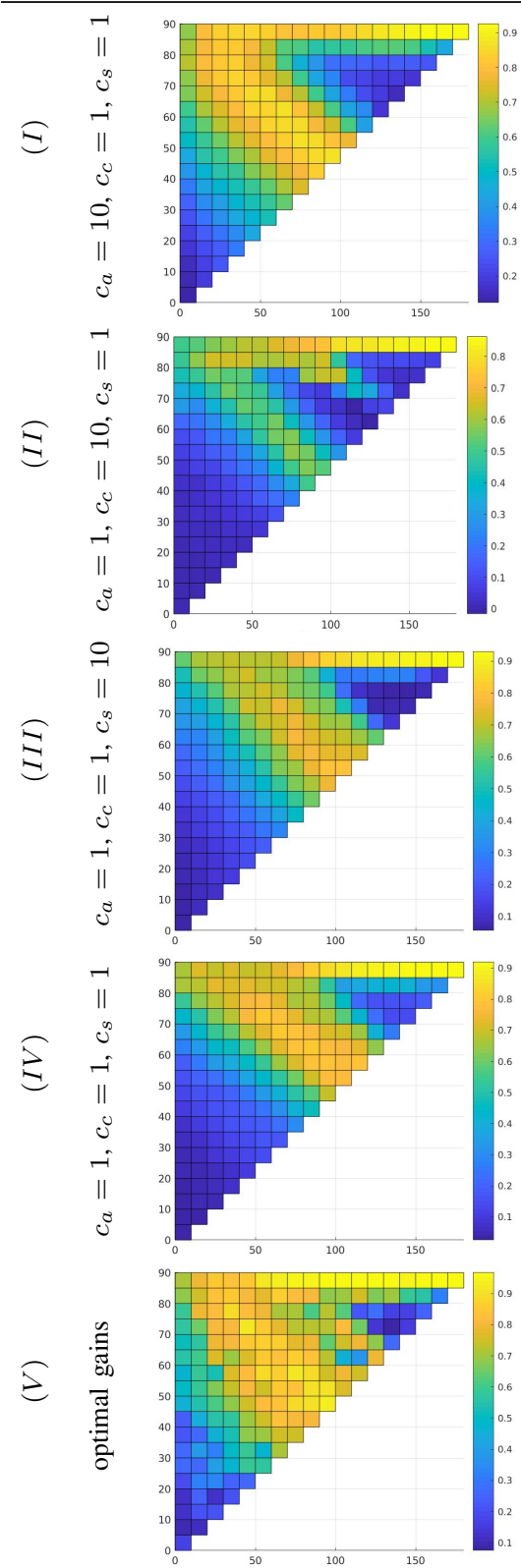

(B) $\Phi_{\text {safety }}$
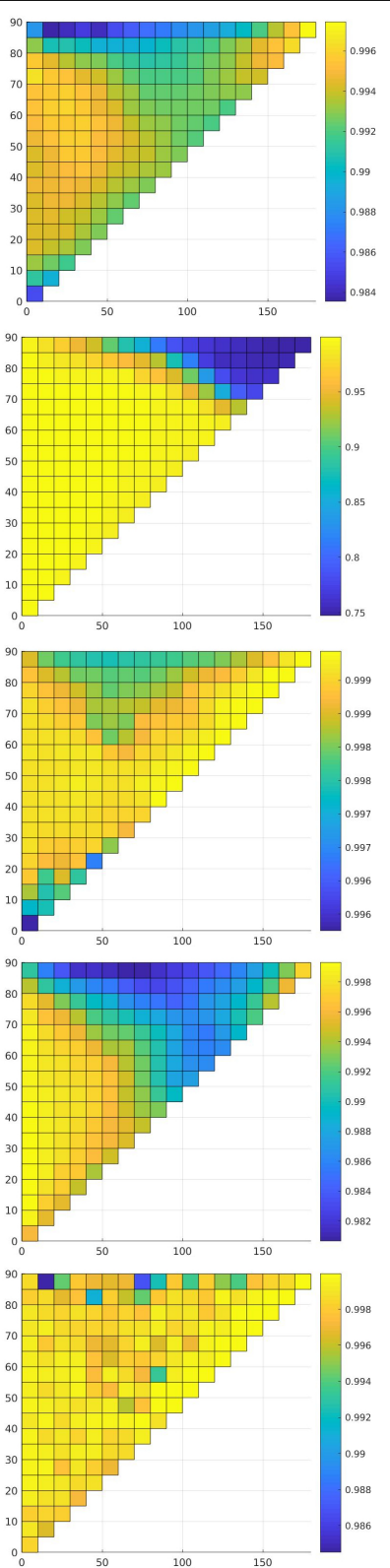

$(C) \Phi_{\text {union }}$
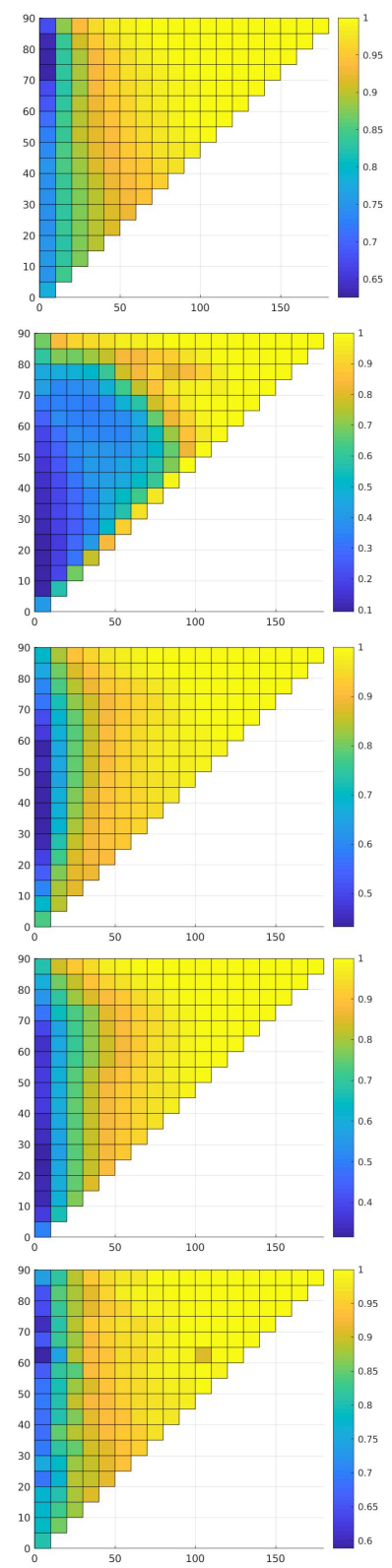

(D) $\Phi_{\text {connectivity }}$
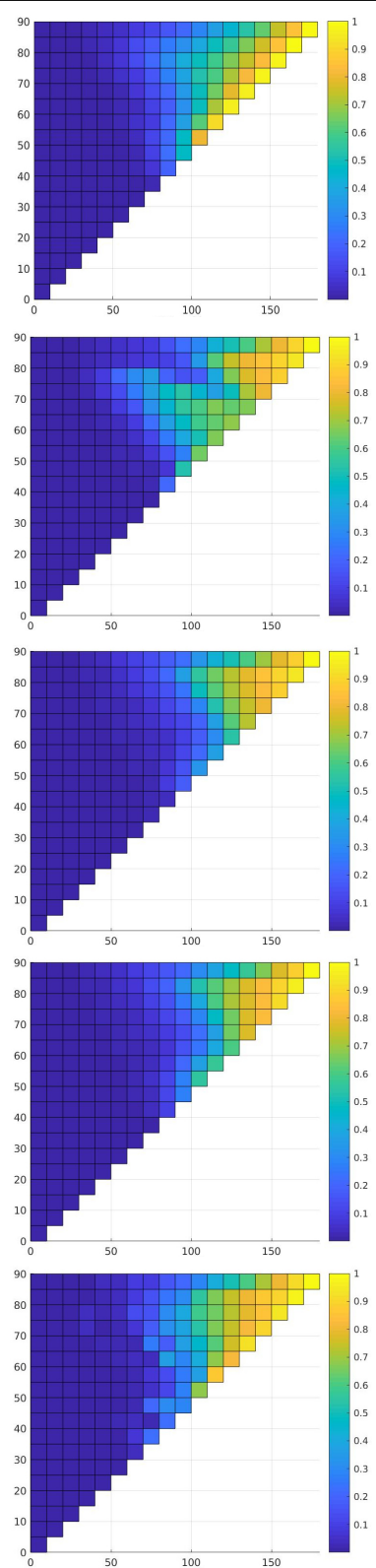

TABLE III: Numerical results of the flocking performance when limited FOV is applied. Every row is associated with a triplet of the Reynolds gains, whose values are specified in the first column. The columns are referred to the different performance metrics. Namely, from left to right, they are the order $\left(\Phi_{\text {order }}\right)$, safety $\left(\Phi_{\text {safety }}\right)$, union $\left(\Phi_{\text {union }}\right)$ and connectivity $\left(\Phi_{\text {connectivity }}\right)$ metrics. The last row $(V)$ shows the results of the flocking performance when the Reynolds gains of every sensor configuration are optimized w.r.t. a given metric. In the plots, the $\mathrm{x}$ axis represents the width angle $\alpha\left[^{\circ}\right]$, varying from $0^{\circ}$ to $180^{\circ}$, while the $\mathrm{y}$ axis represents the azimuth angle $\theta\left[{ }^{\circ}\right]$, varying from $0^{\circ}$ to $90^{\circ}$. Every bin is computed as the average of 100 simulations with randomized initial conditions, i.e. positions and velocities of the flock's agents. Notice that the color-maps are rescaled according to the ranges of the metrics values. 
their precious advice and their technical support all along the production of this paper. This research was partly supported by the Swiss National Science Foundation (SNSF) with grant number 200021155907 and the Swiss National Center of Competence Research (NCCR).

\section{REFERENCES}

[1] K. Karydis and V. Kumar, "Energetics in robotic flight at small scales," Interface Focus, vol. 7, no. 1, 2017.

[2] S.-J. Chung, A. A. Paranjape, P. Dames, S. Shen, and V. Kumar, "A survey on aerial swarm robotics," IEEE Transactions on Robotics, vol. 34, no. 4, pp. 837-855, 2018.

[3] D. Zhou, Z. Wang, and M. Schwager, "Agile coordination and assistive collision avoidance for quadrotor swarms using virtual structures," IEEE Transactions on Robotics, vol. 34, no. 4, pp. 916-923, 2018.

[4] M. Bernard, K. Kondak, I. Maza, and A. Ollero, "Autonomous transportation and deployment with aerial robots for search and rescue missions," Journal of Field Robotics, vol. 28, no. 6, pp. 914-931, 2011.

[5] J. Zhang, J. Hu, J. Lian, Z. Fan, X. Ouyang, and W. Ye, "Seeing the forest from drones: Testing the potential of lightweight drones as a tool for long-term forest monitoring," Biological Conservation, vol. 198, pp. 60-69, 2016.

[6] A. T. Erman, L. van Hoesel, P. Havinga, and J. Wu, "Enabling mobility in heterogeneous wireless sensor networks cooperating with uavs for mission-critical management," IEEE Transactions on Wireless Communications, vol. 15, no. 6, pp. 38-46, 2008.

[7] T. Stirling, J. Roberts, J.-C. Zufferey, and D. Floreano, "Indoor navigation with a swarm of flying robots," in IEEE International Conference on Robotics and Automation, 2012, pp. 4641-4647.

[8] D. Floreano and R. J. Wood, "Science, technology and the future of small autonomous drones," Nature, vol. 521, no. 7553, pp. 460-466, 2015.

[9] D. Mellinger, M. Shomin, N. Michael, and V. Kumar, "Cooperative grasping and transport using multiple quadrotors," in Distributed autonomous robotic systems, 2013 , pp. 545-558.

[10] A. Kushleyev, D. Mellinger, C. Powers, and V. Kumar, "Towards a swarm of agile micro quadrotors," Autonomous Robots, vol. 35, no. 4, pp. 287-300, 2013.

[11] A. Weinstein, A. Cho, G. Loianno, and V. Kumar, "Visual Inertial Odometry Swarm: An Autonomous Swarm of Vision-Based Quadrotors," IEEE Robotics and Automation Letters, vol. 3, no. 3, pp. 1801$1807,2018$.

[12] S. Jha, R. G. Casey-Ford, J. S. Pedersen, T. G. Platt, R. Cervo, D. C. Queller, and J. E. Strassmann, "The queen is not a pacemaker in the small-colony wasps Polistes instabilis and P. dominulus," Animal Behaviour, vol. 71, no. 5, pp. 1197-1203, May 2006.

[13] G. Dell'Ariccia, G. Dell'Omo, D. P. Wolfer, and H.-P. Lipp, "Flock flying improves pigeons' homing: GPS track analysis of individual flyers versus small groups," Animal Behaviour, vol. 76, no. 4, pp. 1165-1172, 2008.

[14] I. D. Couzin, "Collective cognition in animal groups," Trends in Cognitive Sciences, vol. 13, no. 1, pp. 36-43, 2009.

[15] M. Nagy, Z. Ákos, D. Biro, and T. Vicsek, "Hierarchical group dynamics in pigeon flocks," Nature, vol. 464, no. 7290, pp. 890-893, 2010.

[16] H.-T. Zhang, Z. Chen, T. Vicsek, G. Feng, L. Sun, R. Su, and T. Zhou, "Route-dependent switch between hierarchical and egalitarian strategies in pigeon flocks," Scientific Reports, vol. 4, no. 1, 2015.

[17] M. Yomosa, T. Mizuguchi, G. Vásárhelyi, and M. Nagy, "Coordinated Behaviour in Pigeon Flocks," PLOS ONE, vol. 10, no. 10, p. e0140558, 2015.

[18] A. Strandburg-Peshkin, D. R. Farine, I. D. Couzin, and M. C. Crofoot, "Shared decision-making drives collective movement in wild baboons," Science, vol. 348, no. 6241, pp. 1358-1361, 2015.

[19] C. W. Reynolds, "Flocks, Herds, and Schools: A Distributed Behavioral Model," Computer Graphics, p. 21, 1987.

[20] S. Hauert, S. Leven, M. Varga, F. Ruini, A. Cangelosi, J.-C. Zufferey, and D. Floreano, "Reynolds flocking in reality with fixed-wing robots: communication range vs. maximum turning rate," in IEEE/RSJ International Conference on Intelligent Robots and Systems, 2011, pp. 5015-5020.

[21] F. Schilling, J. Lecoeur, F. Schiano, and D. Floreano, "Learning vision-based cohesive flight in drone swarms," arXiv preprint arXiv:1809.00543, 2018.
[22] M. Saska, T. Baca, J. Thomas, J. Chudoba, L. Preucil, T. Krajnik, J. Faigl, G. Loianno, and V. Kumar, "System for deployment of groups of unmanned micro aerial vehicles in gps-denied environments using onboard visual relative localization," Autonomous Robots, vol. 41, no. 4, pp. 919-944, 2017.

[23] G. Vásárhelyi, C. Virágh, G. Somorjai, T. Nepusz, A. E. Eiben, and T. Vicsek, "Optimized flocking of autonomous drones in confined environments," Science Robotics, vol. 3, no. 20, p. eaat3536, 2018.

[24] T. Nestmeyer, P. R. Giordano, H. H. Bülthoff, and A. Franchi, "Decentralized simultaneous multi-target exploration using a connected network of multiple robots," Autonomous Robots, vol. 41, no. 4, pp. 989-1011, 2017.

[25] P. Robuffo Giordano, A. Franchi, C. Secchi, and H. H. Bülthoff, "A passivity-based decentralized strategy for generalized connectivity maintenance," The International Journal of Robotics Research, vol. 32, no. 3, pp. 299-323, 2013.

[26] L. E. Parker, "Distributed algorithms for multi-robot observation of multiple moving targets," Autonomous Robots, vol. 12, no. 3, pp. 231$255,2002$.

[27] G. R. Martin, "Understanding bird collisions with man-made objects: A sensory ecology approach: Bird collisions," Ibis, vol. 153, no. 2, pp. 239-254, 2011

[28] R. Kern, N. Boeddeker, L. Dittmar, and M. Egelhaaf, "Blowfly flight characteristics are shaped by environmental features and controlled by optic flow information," Journal of Experimental Biology, vol. 215, no. 14 , pp. 2501-2514, 2012.

[29] A. Strandburg-Peshkin, C. R. Twomey, N. W. F. Bode, A. B. Kao, Y. Katz, C. C. Ioannou, S. B. Rosenthal, C. J. Torney, H. S. Wu, S. A. Levin, and I. D. Couzin, "Visual sensory networks and effective information transfer in animal groups," Current Biology, vol. 23, no. 17, pp. R709-R711, 2013, 00144.

[30] N. Linander, M. Dacke, and E. Baird, "Bumblebees measure optic flow for position and speed control flexibly within the frontal visual field," Journal of Experimental Biology, vol. 218, no. 7, pp. 1051-1059, 2015.

[31] F. Schiano and P. Robuffo Giordano, "Bearing Rigidity Maintenance for Formations of Quadrotor UAVs," in IEEE International Conference on Robotics and Automation, 2017.

[32] M. Fiedler, "Laplacian of graphs and algebraic connectivity," Banach Center Publications, vol. 25, no. 1, pp. 57-70, 1989.

[33] G. R. Martin, "Visual fields and their functions in birds," Journal of Ornithology, vol. 148, no. S2, pp. 547-562, Dec. 2007.

[34] H. G. Tanner, A. Jadbabaie, and G. J. Pappas, "Stable Flocking of Mobile Agents, Part I: Fixed Topology," IEEE International Conference on Decision and Control, vol. 2, pp. 2010-2015, 2003.

[35] U. Mehmood, N. Paoletti, D. Phan, R. Grosu, S. Lin, S. D. Stoller, A. Tiwari, J. Yang, and S. A. Smolka, "Declarative vs Rule-based Control for Flocking Dynamics," in IEEE/ACM International Symposium on Applied Computing, 2018, pp. 816-823. 